\title{
Influence of Elastic Deformation in Surface Acoustic Wave Motor Friction Drive
}

\author{
Minoru Kuribayashi Kurosawa*, Hidenori Itoh*, and Katsuhiko Asai** \\ *Tokyo Institute of Technology, Dept. of Advanced Applied Electronics, \\ 4259 Nagatsuta, Midori-ku, Yokohama 226-8502, Japan, mkur@ae.titech.ac.jp \\ **Matsushita Electric Industrial Co., Ltd., Advanced Technology Research Laboratories, \\ 3-10-1 Higashimita, Tama-ku, Kawasaki 214-8501, Japan
}

\begin{abstract}
SUMMARY
Importance of elastic deformation control to obtain large output force is discussed. By adding pre-load to slider, stator and slider surfaces are deformed in tens nano meter. Appropriate deformation in normal direction against normal vibration displacement amplitude of surface acoustic wave existed. By moderate deformation, maximum no-load speed was $0.97 \mathrm{~m} / \mathrm{sec}$ which was $88 \%$ of the vibration velocity. The maximum output force was enlarged up to about $10 \mathrm{~N}$. To produce this performance, the transducer weight and slider size were only $4.2 \mathrm{~g}$ and $4 \times 4 \mathrm{~mm}^{2}$. The elastic deformation of the stator was almost same as the vibration displacement amplitude.
\end{abstract}

Keywords: linear motor, friction drive, surface acoustic wave

\section{INTRODUCTION}

We have proposed to utilize HF frequency band (3$30 \mathrm{MHz}$ ) surface acoustic wave (SAW) devices for ultrasonic motors and demonstrated its possibility [1]. A multi contact type slider using a lot of steel balls [2] and a silicon slider [3] have been proposed. They were succeeded in gaining large output force. The driving frequency of SAW device was enhanced up to $70 \mathrm{MHz}$ for miniaturization [4]. For saving the driving electric power, energy circulation driving methods were also investigated [5]. A simple simulation model was proposed to explain excessive

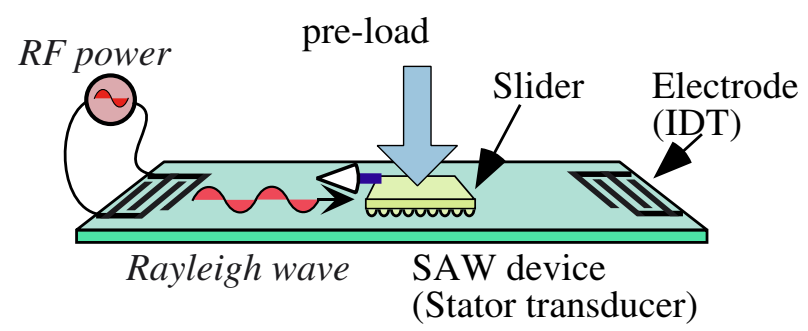

Fig. 1 Schematic view of a surface acoustic wave motor. pre-load causes lower output force [6]. Diameter and density of projections fabricated on silicon sliders were examined experimentally [7].

\section{POINT OF DISCUSSION}

A schematic view of the surface acoustic wave motor is illustrated in Fig. 1. RF electrical power $(9.6 \mathrm{MHz})$ is transduced to elastic wave. The Rayleigh wave is excited at the interdigital transducer (IDT) with piezoelectric effect. By traveling wave propagation, the surface particles of the SAW device move in elliptical motion. Since the amplitude of the elliptical motion is $10 \mathrm{~nm}$ order, the contact condition of the slider is very critical. To control the contact condition, namely, the elastic deformation of the slider and the stator surface in nano meter order, a lot of projections are fabricated on the slider surface. The projection
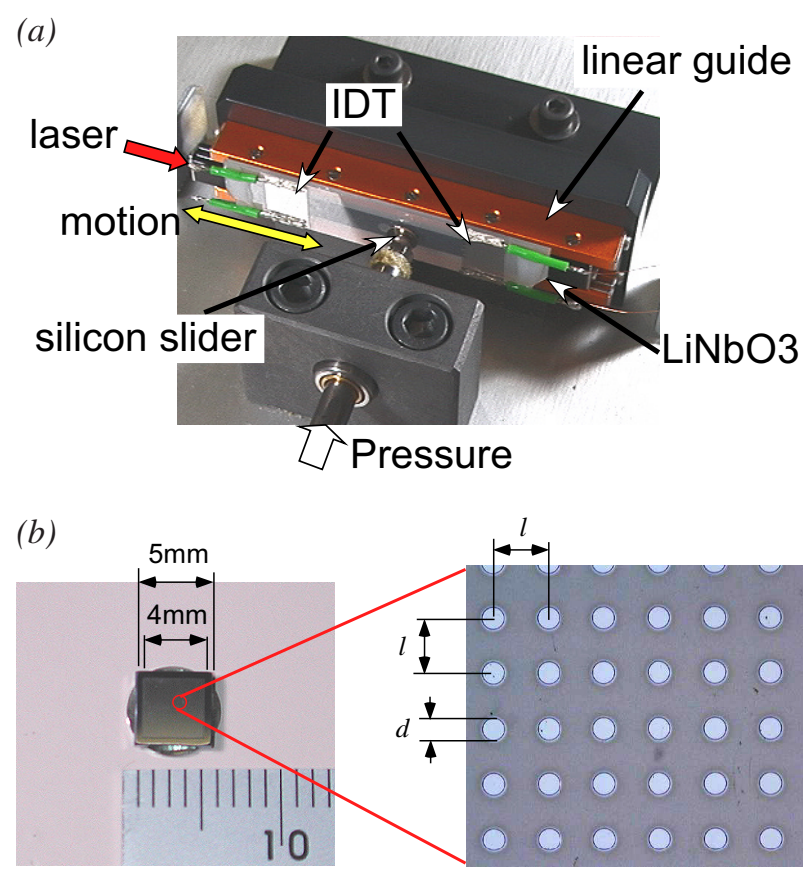

Fig. 2 Photographs of the experimental setup (a) and the silicon slider with enhanced surface profile $(b)$. 
diameter was $20 \mu \mathrm{m}$. An experimental setup and a silicon slider are shown in Fig. 2.

In static condition, the elastic deformation and stress were evaluated with Hertz contact model in case of a single steel ball slider [8]. From this calculation and the simulation result [6], the friction drive mechanism of surface acoustic wave is illustrated as shown in Fig. 3. The wave crest is distorted, hence the elasticity has influence on the friction drive condition.

\section{STATIC ANALYSIS USING THE FEM}

Elastic deformation of the stator surface beneath the projection from the initial position were evaluated with the FEM. An analysis model of the FEM is illustrated in Fig. 4. A pair of cubic parts of the stator and the slider including 4 quarters of projections were taken out. In X and Y direction, the cubic parts had infinite periodic structure. The bottom of the stator was the fixed boundary condition. Static pressure was provided on the silicon slider. Then, the elastic deformation and the stress were evaluated.

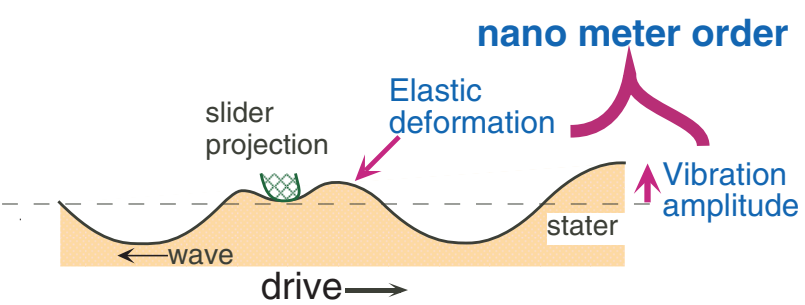

Fig. 3 Image of the elastic deformation of the surface acoustic wave crest due to pre-load.

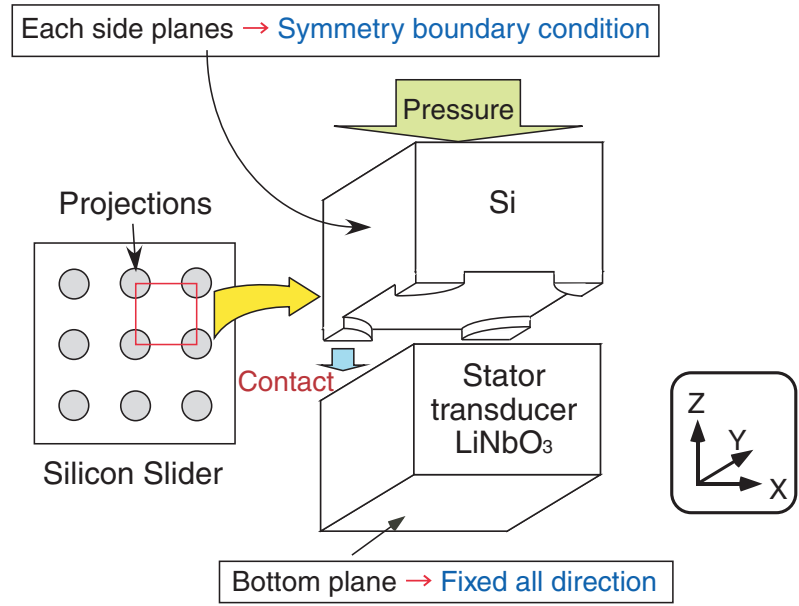

Fig. 4 Unit of the analysis model for the FEM and the boundary conditions.
An example of stress distribution is shown in Fig. 5. Concentration of the stress is observed due to the sharp edge geometry of the projections. The deformation of the stator surface is indicated in Fig. 6 . Beneath the projection, the depression was maximum.

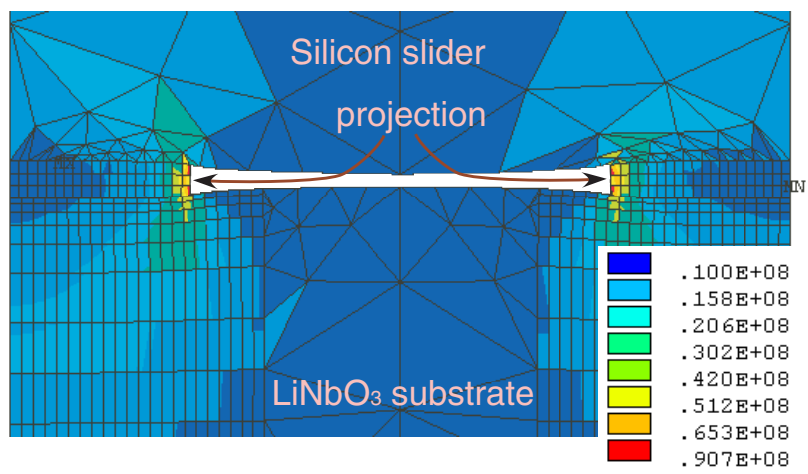

Fig. 5 Stress distribution of stator, slider and slider projection due to pre-load by the FEM analysis. Two half projection and their space are indicated; projection radius is $10 \mu \mathrm{m}$ and projection space is $20 \mu \mathrm{m}$.

(a)

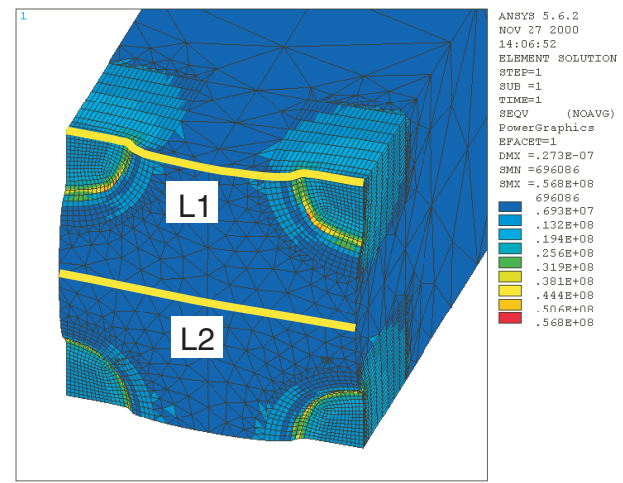

(b)

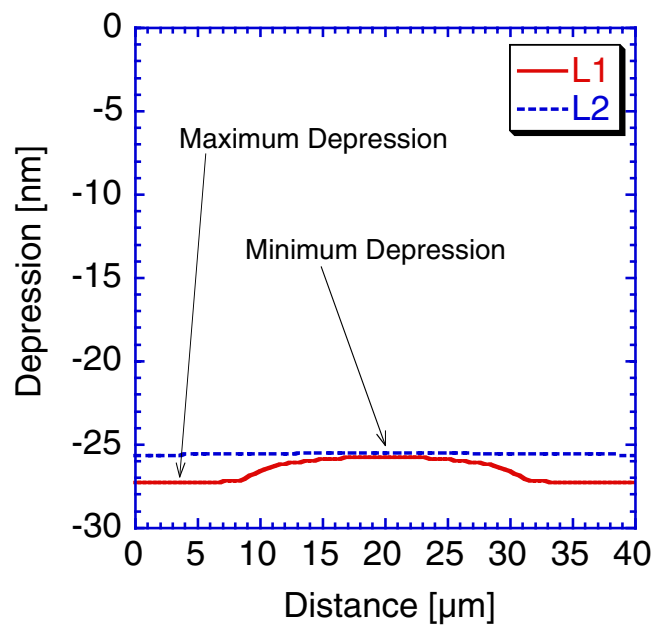

Fig. 6 Analytical results at $88 \mathrm{~N}$ pre-load: (a) stress distribution at contact surface of the stator transducer and (b) depression at the lines L1 and L2. 
The maximum depression changed in proportion to the pre-load and contact pressure as shown in Figs. 7 and 8 . In $4 \times 4 \mathrm{~mm}^{2}$ square area, the sliders had projections from 1089 to 23409 . Depression value of the stator surface were $30 \mathrm{~nm}$ to $50 \mathrm{~nm}$ against $100 \mathrm{~N}$ pre-load. In case of few projections sliders, maximum depression and contact pressure were large. It should be pointed out that the depression become larger than the stator vibration displacement of $20 \mathrm{~nm}$ in normal direction. The other point is low contact pressure. Against $20 \mathrm{~nm}$ maximum depression, the contact pressure were less than 150 $\mathrm{MPa}$. Mostly they are less than $50 \mathrm{MPa}$. The contact pressure of the steel ball sliders were $1 \mathrm{GPa}$ at the maximum.

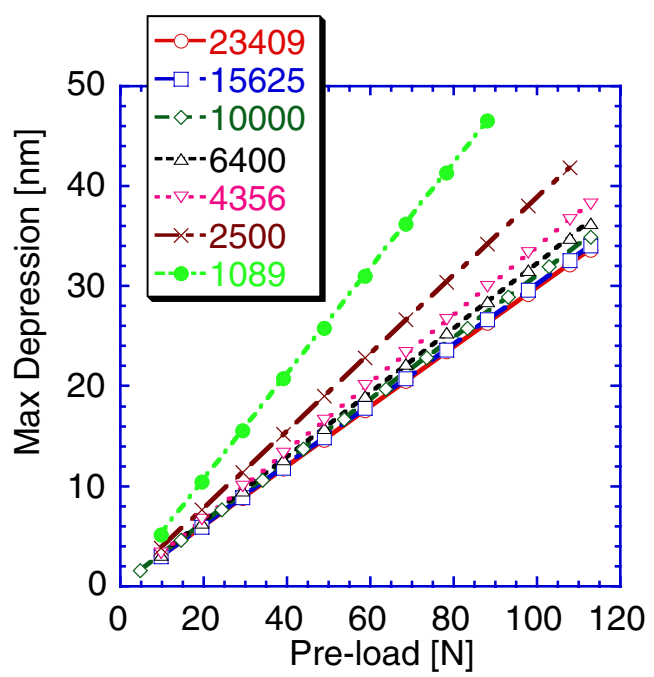

Fig. 7 Maximum depression against pre-load about 7 kinds of sliders which have same projection diameter of $20 \mu \mathrm{m}$ as a function of projection numbers from 1089 to 23409.

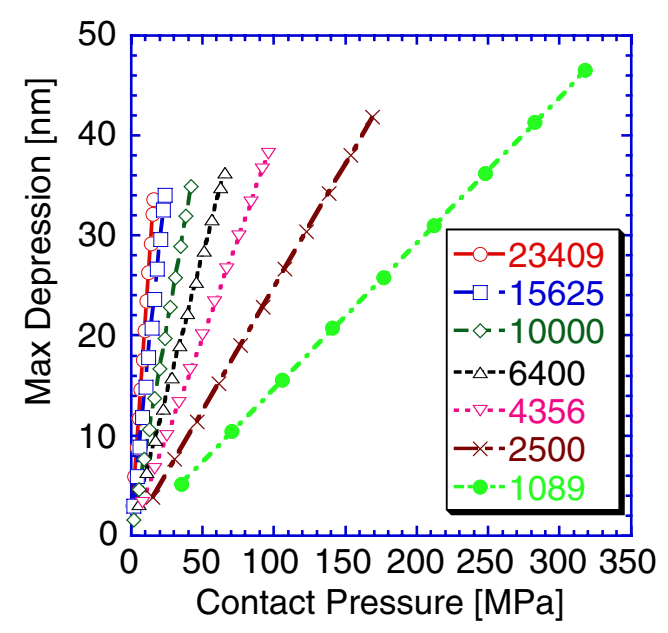

Fig. 8 Maximum depression against contact pressure about 7 kinds of sliders which have same projection diameter of $20 \mu \mathrm{m}$ as a function of projection number.

\section{DRIVING PERFORMANCE}

Mechanical output of the motor such as no-load speed and output force were measured at the driving voltage of $125 \mathrm{~V}_{\text {o-p }}$. At this condition, the vibration amplitude in normal to the stator surface was $21 \mathrm{~nm}$. The preload was changed up to $110 \mathrm{~N}$. Hence, the maximum depression was changed up to about 2 times of the vibration amplitude. Due to the limited space, we indicate only the case of $20 \mu \mathrm{m}$ diameter projection sliders. However, the results of the different diameter projection sliders were similar except for the maximum output force. In lager diameter cases, the maximum output force decreased.

No-load speed were plotted against the ratio of the maximum depression to the vibration displacement amplitude as shown in Fig. 9. No-load speed decreases with increase of the maximum depression, namely, increase of the pre-load. At low pre-load condition below the ratio of 0.5 , the speed saturated. The vibration velocity of horizontal direction was 1.1 $\mathrm{m} / \mathrm{sec}$. The maximum speed was $88 \%$ of the vibration velocity using 23409 projections slider. Influence of the mechanical resistance of the setup, the "no-load speed" seemed to be decreased at small depression. This effect was larger for fewer projections sliders, since the pre-load was smaller. To obtain faster speed, higher density projections slider was superior. This is because larger pre-load is available for many projections slider without increase of elastic deformation.

The output force depended on the depression in spite of the projection density were different as shown in

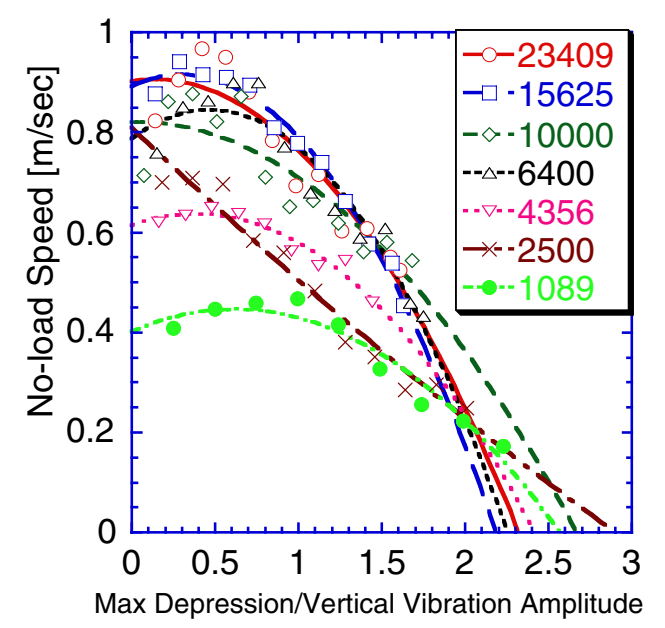

Fig. 9 No-load speed against the maximum depression of the stator surface as a function of the projection numbers of the slider; projection diameter was $20 \mu \mathrm{m}$. 
Fig. 10. The maximum output force was obtained when the maximum depression was from 1.0 to 1.5. This phenomenon was followed by different vibration amplitude. The maximum output force was $10 \mathrm{~N}$ when the highest projection density slider was tested.

The output mechanical power was estimated from the no-load speed and the output force. From the view point of the output power of the motor, the proper maximum depression was also independent to the projection density. The ratio of the depression to the vibration amplitude was almost same as vibration amplitude, namely, the ratio was around 1.0, the output power were maximized.

Different diameter size sliders were tested. The diameters were from $5 \mu \mathrm{m}$ to $50 \mu \mathrm{m}$. These sliders showed similar characteristics as $20 \mu \mathrm{m}$ diameter sliders. Namely, the maximum output force was obtained when the maximum depression was almost same as vibration amplitude.

\section{CONCLUSION}

The relation between the statice elastic deformation by the pre-load and the mechanical output of the motor was examined. We found that the elastic deformation of the whole slider area have influence on the friction drive of the surface acoustic wave motor. Although the local deformation was the point under discussion for the single and the multi-contactpoint sliders using small steel ball(s) [2][8]. The contact pressure was much smaller than that of the steel ball sliders in case of the silicon sliders. We will improve the friction drive model [6] to represent the experimental results discussed here.

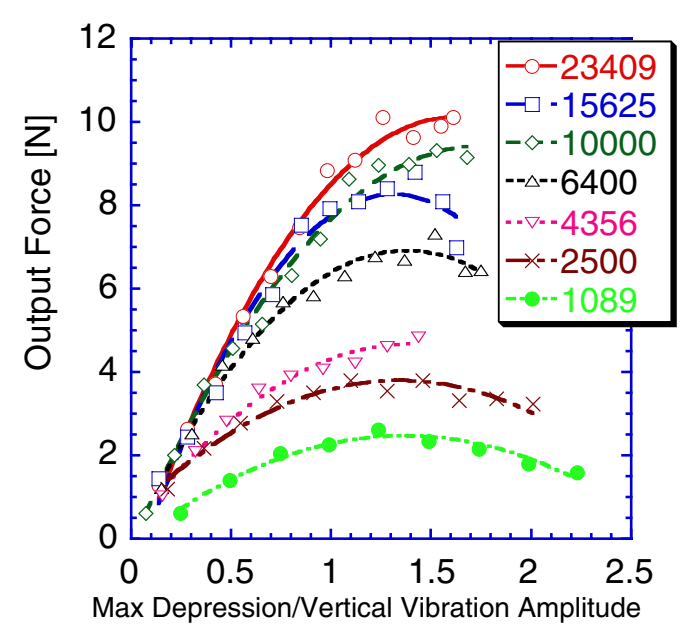

Fig. 10 Output force against the maximum depression of the stator surface as a function of the projection numbers of the slider; projection diameter was $20 \mu \mathrm{m}$.

\section{ACKNOWLEDGMENTS}

This work was supported by the Ministry of Education, Culture, Sports, Science and Technology of Japan.

\section{REFERENCES}

[1] M. Kurosawa, M. Takahashi and T. Higuchi, "Ultrasonic linear motor using surface acoustic wave," IEEE UFFC 43(5), pp. 901-906, 1996.

[2] M. Kurosawa, M. Chiba, and T. Higuchi, "Multi Contact Points Slider for A Surface Acoustic Wave Motor," Trans of Inst. Electrical Engineers of Jpn. E, vol.117E, no.8, pp.430-431, 1997

[3] N. Osakabe, M. Kurosawa, T. Higuchi and O. Shinoura, "Surface acoustic wave linear motor using silicon slider,” MEMS ‘98, pp. 390-395, 1998.

[4] M. Takasaki, M. K. Kurosawa and T. Higuchi, "Optimum silicon slider design for $50 \mathrm{MHz}$ SAW linear motor,' Transducer ‘99, pp. 1756-1757, 1999.

[5] K. Asai, M. K. Kurosawa and T. Higuchi, "Novel power circulation methods for a surface acoustic wave motor,' Proc. of IEEE Ultrason. Symp. '99, pp. 667-670, 1999.

[6] K. Asai, M. K. Kurosawa and T. Higuchi, "Evaluation of the driving performance of a surface acoustic wave linear motor," Proc. of IEEE Ultrason. Symp. 2000, 3C-6 (to be published).

[7] M. K. Kurosawa, H. Itoh, K. Asai, M. Takasaki and T. Higuchi, "Optimization of slider contact face geometry for surface acoustic wave motor," Proc. of MEMS 2001 pp. 252-255, Jan. 21-25, 2001.

[8] M. K. Kurosawa, M. Takahashi and T. Higuchi, "Elastic Contact Conditions to Optimize Friction Drive of Surface Acoustic Wave Motor," IEEE Trans. UFFC, Sept., vol. 45, no. 5, pp. 1229-1237, 1998

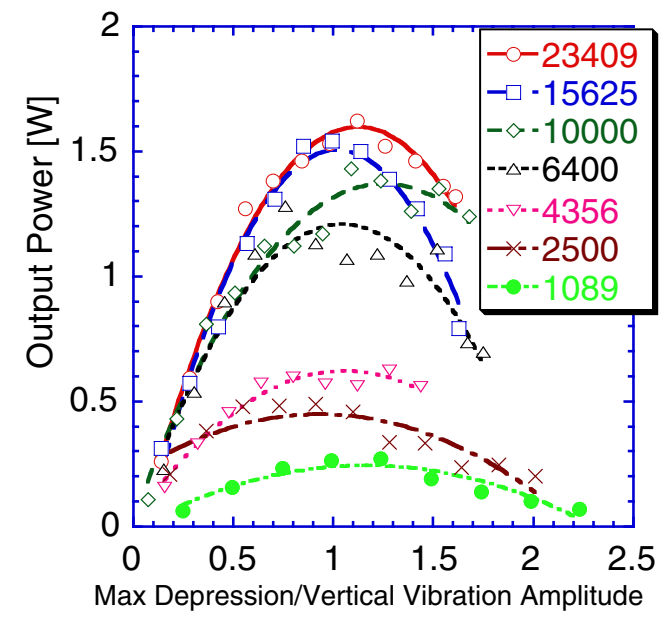

Fig. 11 Output power against the maximum depression of the stator surface as a function of the projection numbers of the slider; projection diameter was $20 \mu \mathrm{m}$. 